\title{
Radial transfer of tracking data with wireless links
}

\author{
Daniel Pelikan ${ }^{* t}$ \\ Uppsala University (SE) \\ E-mail: daniel.pelikan@cern.ch
}

\section{Nils Bingefors}

Uppsala University (SE)

E-mail: nils.bingeforsephysics.uu.se

\section{Richard Brenner}

Uppsala University (SE)

E-mail: Richard.Brenner@cern.ch

\section{Dragos Dancila}

Uppsala University (SE)

E-mail: dragos.dancilaeangstrom.uu.se

\section{Leif Gustafsson}

Uppsala University (SE)

E-mail: leif.gustafsson@physics.uu.se

Wireless data transfer has revolutionized the consumer market for the last decade giving products equipped with transmitters and receiver for wireless data transfer. Wireless technology has features attractive for data transfer in future tracking detectors. The removal of wires and connectors for data links is certainly beneficial both for the material budget and the reliability of the system. One other advantage is the freedom of routing signals which today is particularly complicated when bringing the data the first $50 \mathrm{~cm}$ outside the tracker. With wireless links intelligence can be built into a tracker by introducing communication between tracking layers within a Region Of Interest which would allow the construction of track primitives in real time. The wireless signal is transmitted by a passive antenna structure which is a radiation hard and much less complex object than an optical link. Due to the requirement of high data rates in detectors a high bandwidth is required. The frequency band around $60 \mathrm{GHz}$ has large potential for use in tracking detectors. The high baseband frequency allow for data transfer of the order of several Gbit/s, and due to the small wave length in the mm range, only small structures are needed. One challenge in applying the technology to trackers is to bring the signal around or through boundaries that are not transparent to the mm-waves, i.e. silicon detector modules or support structures. This article presents a test setup of a $60 \mathrm{GHz}$ data link for radial transmission of mm-waves through an ATLAS detector model using a passive repeater structure. We demonstrate that by using a passive repeater structure made of two $60 \mathrm{GHz}$ patch array antennas connected by a micro strip we can make the signal pass boundaries.

Technology and Instrumentation in Particle Physics 2014

2-6 June, 2014

Amsterdam, the Netherlands

\footnotetext{
*Speaker.

†Corresponding author
} 


\section{Introduction}

\subsection{Wireless technology}

Wireless data transfer is becoming a common technology. People connect to the internet using wireless technologies like WLAN, UMTS, LTE and many more. The industry is also working on connecting sensors and devices in cars and other products via wireless technology. The more we develop a connected world the more wireless connections we will use. On the other side, the amount of data being transferred is increasing. The named examples are using frequencies up to a couple of GHz. The more bandwidth needed to cope with the increasing amount of data can be handled by using more advanced modulation techniques or by going to even higher frequency bands. If one wants to go to data rates of several Gbit/s, which one would need e.g. in a track trigger, one needs a lot of bandwidth. The lower $\mathrm{GHz}$ frequency bands simply do not offer enough data transfer capacity if one respects the frequency regulations on these bands. In the $60 \mathrm{GHz}$ frequency band up to $7 \mathrm{GHz}$ unlicensed frequency are available [1] for data transfer. This provides an enormous amount of bandwidth.

The high frequencies bring some disadvantages, at least when looking from the consumer market point of view. The absorption of high frequencies in the transmission medium increases and transfers through walls as required for WLAN are not possible. That is also why the $60 \mathrm{GHz}$ frequency band is more likely to be used for short distance connections, like connecting a mobile phone with a television set and transferring uncompressed HD video to the screen. In the last years industry has developed many of new products using the $60 \mathrm{GHz}$ frequency band and the first products are becoming commercially available.

When bringing the $60 \mathrm{GHz}$ technology into a detector system the short range can actually be an advantage since frequencies can easier be reused. Also, going to higher frequencies makes the wave length shorter and with this the structure sizes of the electronic components. At $60 \mathrm{GHz}$, the wavelength is of the order of $5 \mathrm{~mm}$, thus the term of mm-waves. This leads to very small components, using little space and power [6] which is beneficial when thinking of implementing them in a particle detector. Another important point, when transmitting over short distances, is that the receiving antenna needs to be outside the near-field of the sending antenna.

\subsection{Antenna design}

For the integration of wireless technology in the detector system one of the key components is the antenna which can easily be integrated into the detector electronics and will not add much in material. A very good forward gain and focus would be beneficial in order to reduce the power consumption needed to feed the antenna, but also to reduce cross talk. One type of antenna simple to fabricate and widely used is the patch antenna [3]. This antenna can be directly produced on PCB material, has a flat structure and can be designed to give high focus and gain. This type of antennas can be found in mobile phone applications, wireless LAN, radar and other radio applications. An advantage of this type of antennas is that it is robust against mechanical damage.

\subsubsection{Simulation}

In order to optimize antenna designs and to study their properties the High Frequency Structure Simulation program (HFSS) [4] was used. With the simulation, dimensions for fabrication were 
derived and studies were performed in order to see the forming of the radiation direction and beam forming properties. Also studied was the resonance frequency and the bandwidth of the antenna. Single patch and multi patch antennas were designed. The simulation was used to match the antenna to the design resonance frequency and to match to $50 \Omega$ impedance. Pictures of the simulation design and the beam forming are shown in figure 1.

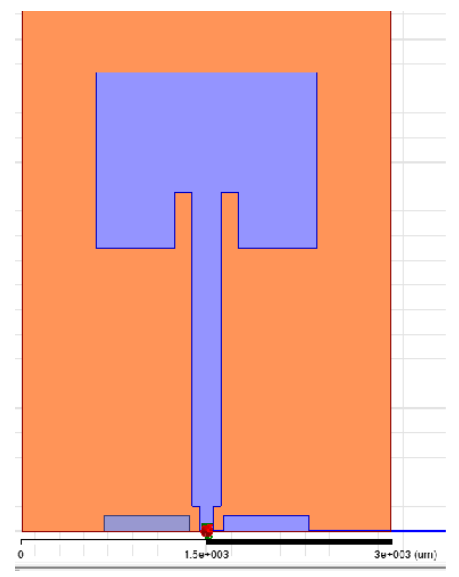

(a) Single patch antenna.

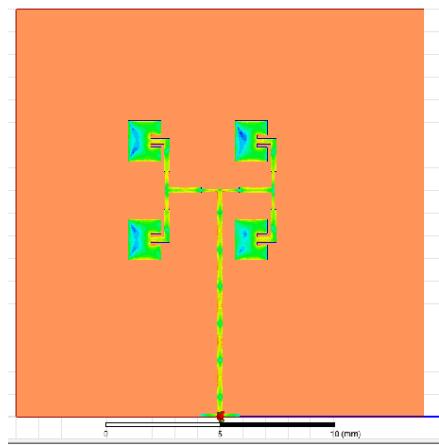

(c) Four patch array antenna.

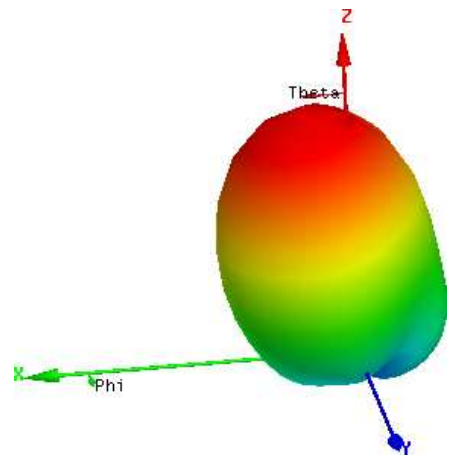

(b) Far field radiation cone of a single patch antenna.

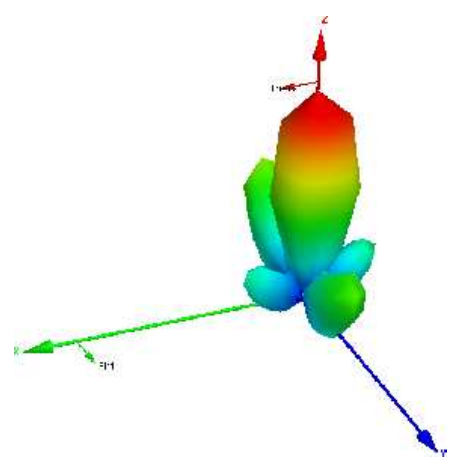

(d) Far field radiation cone of a four patch array antenna.

Figure 1: Simulation model of a single patch antenna and four patch antenna array (a) and (c). The plot (b) and (d) shows the radiation cone of the two antennas. A clear improvement of the radiation direction can be seen. The four patch array is more focused in the forward direction compared to the single patch. This leads to a higher gain on this antenna.

\section{Wireless data transfer through a particle detector using a passive repeater structure}

Radial transfer of data in trackers could bring many advantages to the design and reduce the amount of electronics significantly. Data links could be spread uniformly around a detector and would not have to be routed through extreme dense gaps like in ATLAS [2] in the crack region at $\eta<0.8$. An even better solution would be, if no active repeater structure is be needed [5]. 


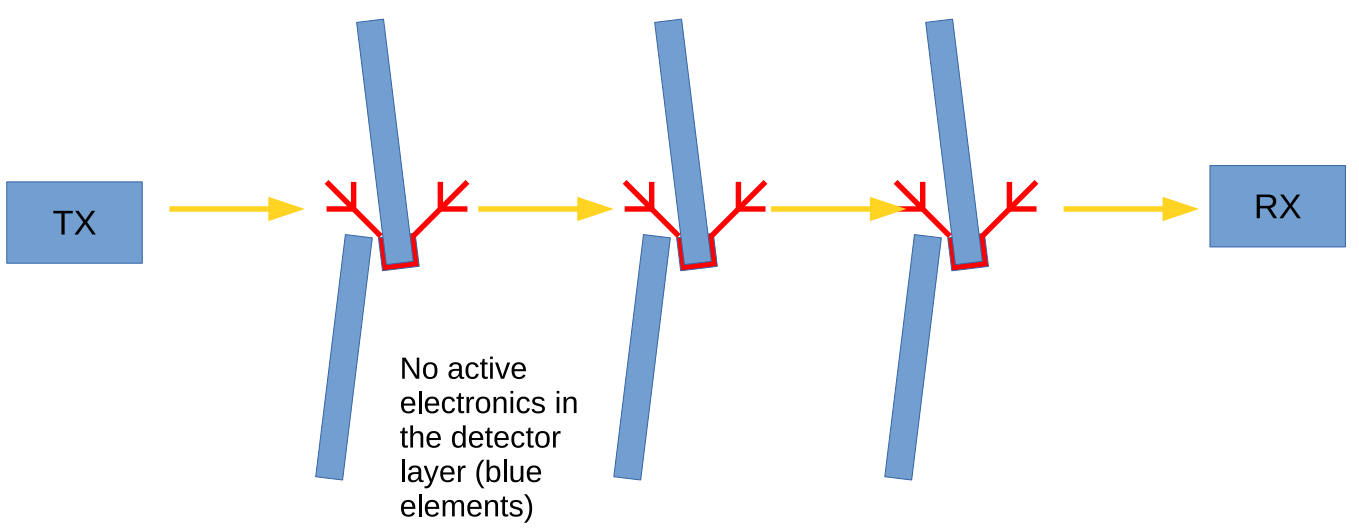

Figure 2: Schematic of a wireless data transfer using a passive repeater structure to bring the data through the detector layers. The transfer starts on the transmitter (TX) side, the radio wave is radiated on the receiving antenna sitting on the first detector layer. The energy collected by the receiving antenna is passed to the other side of the detector layer and is there retransmitted by an antenna. On the second layer the energy is collected again and retransmitted on the other side. This continues until the receiver (RX) is reached for further processing. No active electronics are required in the intermediate part. The antennas are just connected by micro strips, through the gaps between the detector elements.

This approach is shown in figure 2. Starting on the right side with a transmitter (TX) which is sending a signal at $60 \mathrm{GHz}$. The signal is focused in the forward direction, radiating the receiver side of the passive antenna structure. From there the signal travels to the other side to the transmitting antenna and is radiating on the next antenna. This is repeated until the actual receiver (RX) is reached. The receiver receives the signal for further processing. No active components except transmitter and receiver are needed in this data transfer.

\subsection{Passive antenna structure}

A passive repeater structure will require two high gain antennas coupled to each other with small transmission losses. This is archived by an array of patch antennas which are connected by micro strips as shown in figure 3. As can be seen, the small micro strips have variations in thickness, which are needed in order to match the impedance between the patches. The repeater structure is of course symmetric, where each antenna array acts as a receiver or transmitter. The antenna array was produced by milling DuPont PCB material AP9161R.

\subsection{The test setup}

Transmission tests with antenna structures were performed in a mock-up of a tracker. The tracker layers were made of aluminium slabs and one or two layers could be positioned between TX and RX. The transmitter and receiver consists of two identical $60 \mathrm{GHz}$ up/down converter cards made by SIVERSIMA, see figure 4 . The cards are duplex cards and have both an RX unit and an TX unit. The data is split in I and Q components that enable testing with different modulation schemes. The cards have been equipped with horn antennas $(10 \mathrm{dBi})$, to focus the beam into the forward direction. A simplified sketch of the whole setup is shown in figure 5.

Starting on the transmitting side, a sine wave signal at $1 \mathrm{GHz}$ is sent from a frequency generator through a power splitter into the I and Q of the converter card. The power splitter is needed in order 


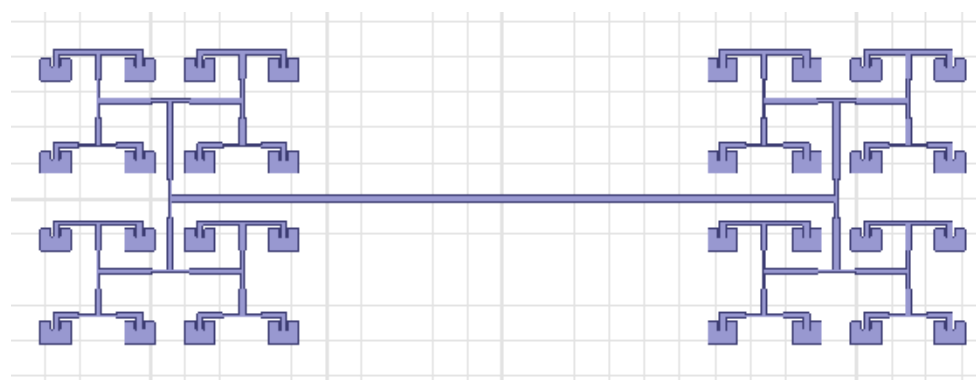

Figure 3: Passive repeater: Two 16 patch antenna arrays are connected by a micro strip. The small micro strips connecting the patches vary in width and act as transformers in order to match the impedance between the patches. The long micro strip connecting the two antenna arrays transports the energy from the receiving side to the transmitting side of the repeater structure. Such a structure can be folded around the edge of a detector element to bring a $60 \mathrm{GHz}$ signal for example from the front to the back.

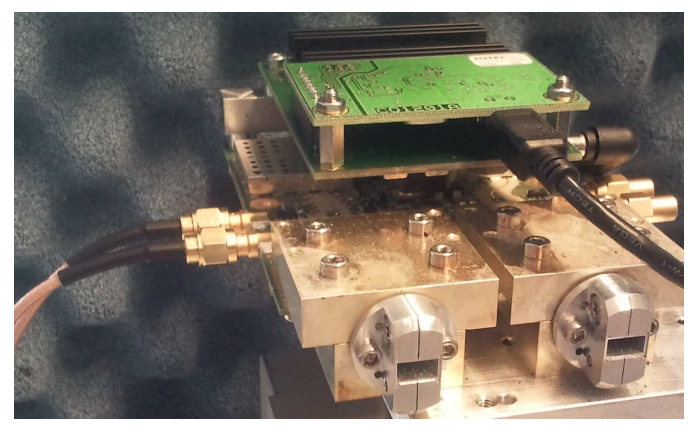

Figure 4: $60 \mathrm{GHz}$ up/down converter card by SIVERSIMA. This duplex card has one up converter to convert from baseband to $60 \mathrm{GHz}$ and one down converter which converts from $60 \mathrm{GHz}$ down to baseband. After up conversion the signal is transmitted via a wave guide. On the down converter side the signal is received from a wave guide. The wave guides can be equipped with horn antennas. I and Q channels are on the receiver and transmitter side brought out separately.

to produce an in phase and a 90 degree phase shifted signal for the I and Q channels of the converter card. In the $60 \mathrm{GHz}$ converter the signal is mixed and up converted. After up conversion the signal is transmitted through the horn antenna. In order to simulate the radiation through the detector system one aluminium slab is placed in front of the horn antenna separating the receiver side from the transmitter side. The metal plate is equipped with a small gap in order to bring through the micro strip connecting the receiver and transmitter side of the passive repeater structure. To make sure that no electro magnetic waves are travelling through the gap, and all energy is transported through the micro strip to the other side, the gap is sealed with metal tape. Additionally, to get rid of reflections each side of the metal plate is covered with absorbing foam with holes for the antenna. The transmitter side radiates on to the antenna of the passive repeater structure, the signal is received and travels along the micro strip to the other side. There the received energy excites the antenna on the other side. The excited antenna radiates the signal which then is received by the down converter. The down converter converts the signal down from $60 \mathrm{GHz}$ to baseband. The I and Q outputs of the down converter are fed through a power combiner into an oscilloscope in order to make the received signal visible. If the signal comes through the passive structure, a peak 


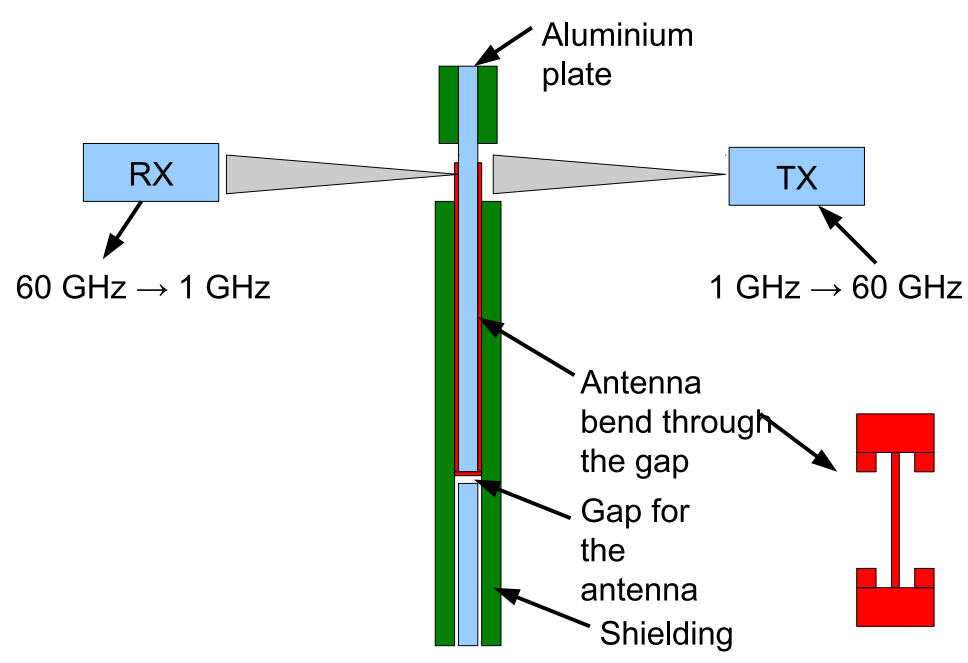

Figure 5: Schematic of one test setup. On the transmitter side (TX) a signal from a frequency generator @ $1 \mathrm{GHz}$ is fed into the up converter and converted to $60 \mathrm{GHz}$. The signal is transmitted as an electro magnetic wave from the sender and is radiated on the receiving patch antenna. This antenna is connected with a micro strip to the other side of a metal plate with another patch antenna acting as sending element. The micro strip is brought to the other side through a small gap. In order to make for the experiment sure that no electromagnetic radiation goes through this small gap, the gap is closed with metal tape. In order to reduce reflections between the sender and the aluminium plate an absorbing foam (shielding) is placed on both sides of the metal plate. Leaving only a hole where the antenna sits. When the signal has travelled through from the receiving antenna through the micro strip to the sending antenna it is received by the receiver (RX) which does a down conversion from $60 \mathrm{GHz}$ to the input frequency. The down converted signal is detected in an oscilloscope.

at the frequency of the signal generator $(1 \mathrm{GHz})$ can be observed as seen in figure 6(b).

To make the test even more realistic in terms of being inside a detector, also an ATLAS inner detector model made of aluminium was used, see figure 6(a). There two layers of detector were equipped with a passive antenna and radiated through both layers. This proves that it is possible to transmit through two layers with passive structures. With more optimized designs it is expected that transfer through even more layers is possible. Also in this test the gaps are closed with metal tape in order to prevent the signal taking any other way than through the micro strip.

\section{Power loss in the detector layers}

One figure of merit for a passive repeater structure is the signal attenuation. This is important in order to keep the total power consumption and cooling low. The described setup in section 2 transmits the signal with $1 \mathrm{~mW}$.

Figure 7 shows different scenarios tested. The first test (black line) was to radiate from the TX side to the RX side using a horn antenna on each side at a distance of $12 \mathrm{~cm}$. A received power of better than $-40 \mathrm{dBm}$ was detected. This is the baseline performance of the testsystem. A slight frequency dependence on the output power can be observed, coming from inefficiencies in the conversion and transmission. The next step was to put one detector layer in between the two horn antennas, equipped with a passive antenna (green line) with 16 patches on each side. It 


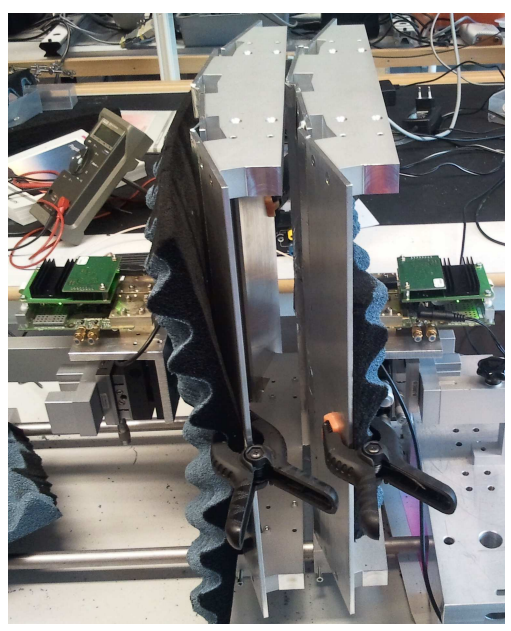

(a) Passive data transfer through a two layer ATLAS inner detector model.

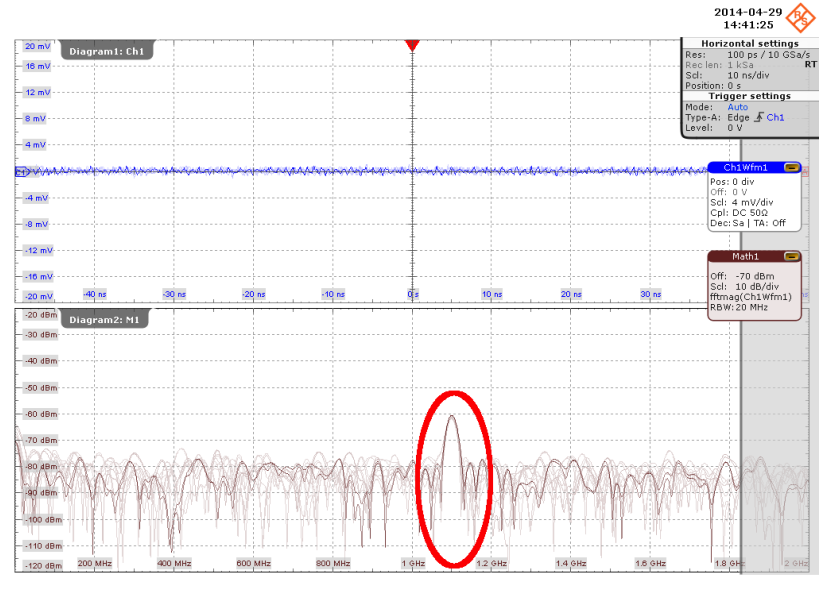

(b) Received signal after passing the passive repeater structure.

Figure 6: Experimental setup of a passive repeater structure (a) and the received signal on the oscilloscope (b). The signal seen in (b) has been sent from the $60 \mathrm{GHz}$ up converter, travelled through two layers of a aluminium ATLAS model and was received and down converted and sent to the oscilloscope. To make sure that no radiation goes through the gaps of the model, they were closed with metal tape.

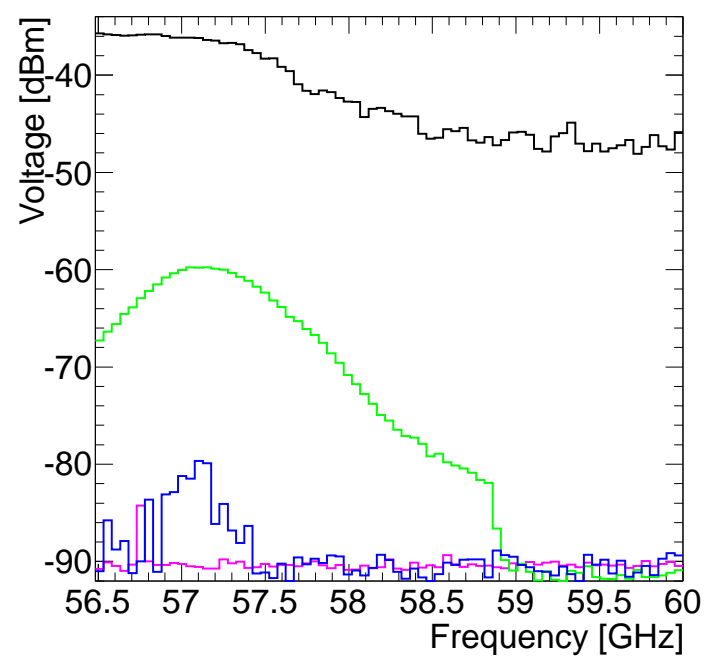

Figure 7: Signal strength of the received signal when sending with $0 \mathrm{dBm}(1 \mathrm{~mW})$ output power. (black) Horn antenna to horn antenna at a distance of $12 \mathrm{~cm}$. The horn antenna has a gain of $10 \mathrm{dBi}$. (green) Horn antenna through passive repeater to horn antenna. (blue) Horn antenna through two layers of passive repeater to horn antenna. (purple) This line represents the background when having a metal plate without antenna between the two horn antennas. 
can be seen that the received power is highly frequency dependent and that the highest point is at around $57.2 \mathrm{GHz}$. The top at $57.2 \mathrm{GHz}$ is the point where the antenna is most resonant and works best. It can also be seen that the highest output is $20 \mathrm{~dB}$ lower compared to the output from horn to horn. This difference represents the attenuation in a single detector layer. The loss comes from the fact that not all power which is transmitted is received, but also from the fact that the antenna itself is absorbing power. Finally a second antenna was placed in the path leading to another drop of around $20 \mathrm{~dB}$ (blue line). For comparison the background line (purple) is shown, representing the received power when a detector layer is placed between transmitter and receiver, but no passive repeater is in place. It is expected that the losses in each detector layer can be reduced by further optimising of the antennas, but also by using materials with a smaller dielectric constant, in order to reduce the losses in the antenna. The study proves that data transfers are possible by using a passive repeater structure and that radial data links can be built.

\section{Conclusion}

The idea of a radial data transfer setup has been described and a test setup has been built and the radial data transfer was demonstrated by transferring a sine wave at $60 \mathrm{GHz}$ from a horn antenna through two layers of a passive repeater structures to a receiving horn antenna. The signal was received by an oscilloscope showing a peak at the baseband frequency. A first estimate of how much power is lost in each layer was performed. With the used 16 patch repeater antenna arrays a loss of approximately $20 \mathrm{~dB}$ per layer is archived. With further improvements on the repeater antennas this value should be possible to decrease. The experimental study showed that the principle of using $60 \mathrm{GHz}$ wireless together with a passive repeater structure is possible and that signals can be transferred through such a system.

\section{Acknowledgments}

The authors want to thank Carl Tryggers foundation for supporting this work.

\section{References}

[1] S. Khiong Yong and C. Chong, An overview of multigigabit wireless through millimeter wave technology: potentials and technical challenges. EURASIP J. Wirel. Commun. Netw. 1 (2007) 50, Article ID 78907

[2] ATLAS Collaboration, ATLAS detector and physics performance: Technical Design Report, 1. CERN, Geneva, 1999

[3] D. M. Pozar and D. H. Schaubert, Microstrip Antennas: The analysis and design of microstrip antennas and arrays. IEEE Press, New York, 1995.

[4] http://www.ansys.com/Products/

[5] R. Brenner and S. Cheng, Multigigabit wireless transfer of trigger data through millimetre wave technology. 2010 JINST 5 C07002, in Workshop on Intelligent Trackers 2010, Lawrence Berkeley National Laboratory, Berkeley U.S.A., 3-5 Feb 2010.

[6] Marcu, C., et al., A 90 nm CMOS Low-Power 60 GHz Transceiver With Integrated Baseband Circuitry. Solid-State Circuits, IEEE Journal of (2009), Volume: 44 , Issue: 12 